\title{
Comprehensive analysis of NAC transcription factors and their expression during fruit spine development in cucumber (Cucumis sativus L.)
}

Xingwang Liu', Ting wang ${ }^{1}$, Ezra Bartholomew ${ }^{1}$, Kezia Black${ }^{1}$, Mingming Dong ${ }^{1}$, Yaqi Zhang ${ }^{1}$, Sen Yang ${ }^{1}$, Yanling Cai ${ }^{1}$, Shudan Xue', Yiqun Weng ${ }^{2}$ and Huazhong Ren ${ }^{1}$

\begin{abstract}
The cucumber (Cucumis sativus L.) is an important vegetable crop worldwide, and fruit trichomes or spines are an important trait for external fruit quality. The mechanisms underlying spine formation are not well understood, but the plant-specific NAC family of transcription factors may play important roles in fruit spine initiation and development. In this study, we conducted a genome-wide survey and identified 91 NAC gene homologs in the cucumber genome. Clustering analysis classified these genes into six subfamilies; each contained a varying number of NAC family members with a similar intron-exon structure and conserved motifs. Quantitative real-time PCR analysis revealed tissue-specific expression patterns of these genes, including 10 and 12 that exhibited preferential expression in the stem and fruit, respectively. Thirteen of the 91 NAC genes showed higher expression in the wild-type plant than in its near-isogenic trichome mutant, suggesting their important roles in fruit spine development. Exogenous application of four plant hormones promoted spine formation and increased spine density on the cucumber fruits; several NAC genes showed differential expression over time in response to phytohormone treatments on cucumber fruit, implying their essential roles in fruit-trichome development. Among the NAC genes identified, 12 were found to be targets of 13 known cucumber micro-RNAs. Collectively, these findings provide a useful resource for further analysis of the interactions between NAC genes and genes underlying trichome organogenesis and development during fruit spine development in cucumber.
\end{abstract}

\section{Introduction}

Transcription regulation of gene expression is an important control point of many biological processes in plant growth and development, as well as responses to biotic and abiotic stresses ${ }^{1-3}$. During this process, transcription factors (TFs) function as regulatory trans-acting

Correspondence: Yiqun Weng (yiqun.weng@ars.usda.gov) or

Huazhong Ren (renhuazhong@cau.edu.cn)

${ }^{1}$ Beijing Key Laboratory of Growth and Developmental Regulation for

Protected Vegetable Crops, College of Horticulture, China Agricultural University, 100193 Beijing, P. R. China

${ }^{2}$ Department of Horticulture, USDA-ARS, Vegetable Crops Research Unit, University of Wisconsin-, Madison, WI 53706, USA

These authors contributed equally: Xingwang Liu and Ting wang elements that bind to specific cis-regulatory elements in the promoters of target genes to activate or repress expression of the target genes ${ }^{4}$. Thus, the identification and functional characterization of TFs is of great importance in understanding the transcriptional regulatory networks controlling different cellular processes ${ }^{5}$. Numerous plant TF families have been identified and characterized according to their DNA-binding motifs. Some notable examples include apetalous (AP2), basic leucine zipper domain (bZIP), myeloblastosis oncogene (MYB), myelocytomatosis oncogene (MYC), Mcm1Agamous-Deficiens-Srf (MADS), homeodomain leucine zipper (HD-Zip), basic helix-loop-helix (bHLH), Cys2/

\section{(c) The Author(s) 2018}

(c) (i) Open Access This article is licensed under a Creative Commons Attribution 4.0 International License, which permits use, sharing, adaptation, distribution and reproduction cc) in any medium or format, as long as you give appropriate credit to the original author(s) and the source, provide a link to the Creative Commons license, and indicate if changes were made. The images or other third party material in this article are included in the article's Creative Commons license, unless indicated otherwise in a credit line to the material. If material is not included in the article's Creative Commons license and your intended use is not permitted by statutory regulation or exceeds the permitted use, you will need to obtain permission directly from the copyright holder. To view a copy of this license, visit http://creativecommons.org/licenses/by/4.0/. 
His2-type zinc finger (C2H2), as well as the NAC (NAM, ATAF1/2, and CUC) transcription factor families ${ }^{6-\overline{8}}$. The $\bar{N} A C$ is among the largest plant-specific TF families. Genome-wide surveys have identified 106 NAC TF members in the Arabidopsis thaliana (Arabidopsis hereinafter) genome, which are 149 in rice (Oryza sativa), 96 in cassava (Manihot esculenta), 167 in banana (Musa acuminata), 74 in grape (Vitis vinifera), 101 in soybean (Glycine max), and 163 in Populus trichocarpa ${ }^{9-17}$.

A typical NAC TF has a conserved NAC domain of $\sim 150$ amino acids at the $\mathrm{N}$-terminus and a more divergent C-terminal transcription regulatory (TR) region ${ }^{18,19}$. The NAC domain contains five subdomains (A-E) that represent motifs for both DNA-binding (DB) and protein-protein interactions ${ }^{20}$. The highly conserved subdomains $C$ and $D$, with net positive charges, bind to specific cis-regulatory DNA sequences, whereas subdomain $\mathrm{A}$ functions in the dimerization of the $\mathrm{TF}^{18,20-22}$, and the diverse subdomains $\mathrm{B}$ and $\mathrm{E}$ may be responsible for the functional diversity of the NAC gene $^{23,24}$. Based on their motifs, the rice NAC proteins (OsNAC) have been classified into 15 types, from $\mathrm{A}$ to $\mathrm{O}$, with types A-E containing five motifs in DB domains, whereas types $\mathrm{F}-\mathrm{O}$ are present in NAC-like proteins with distinct motif compositions ${ }^{25}$. Such motif variations highlight the involvement of NAC TFs in diverse functions. The NAC domain also modulates protein binding, which may determine the fate and functions of the NAC proteins ${ }^{26}$. In some NACs, a highly hydrophobic region in a negative regulatory domain (NRD) may form a part of the $\mathrm{D}$ subunit, which suppresses transcriptional activity of the NAC TFs. The NAC-repressor domain may inhibit the activity of other TF family members such as WRKY and $\mathrm{AP2}{ }^{27}$. The $\mathrm{C}$-terminal TR domain may act as either an activator or a repressor, and sometimes displays protein-binding activity. In Arabidopsis, it was found that the highly divergent TR domain has 13 common motifs in 12 out of the 18 subgroups of the NAC $\mathrm{TF}^{21}$. In addition, an $\alpha$-helical transmembrane motif (named NTLs) is present in some NACs that is required for plasma membrane or endoplasmic reticulum membrane anchoring of the $\mathrm{C}$-terminal region. To date, fewer than 20 NTLs have been identified in any single plant species: 18 NTLs have been found in $A$. thaliana, 11 in soybean, and seven in maize, Zea mays. NTL proteins may play important regulatory roles in response to environmental cues ${ }^{28-31}$.

Since the cloning of the first NAC gene (NAM) from petunia in $1996^{9}$, the NAC TFs have been shown to play important roles in various biological processes, as well as responses to abiotic stresses. For example, in cotton, Gossypium hirsutum, the expression of some NAC genes such as GhNAC22 and GhNAC34 was strongly regulated by salinity and drought stresses, while in maize, natural variation in the NAC gene $Z m N A C 111$ was associated with drought tolerance ${ }^{3,32-34}$. Genome-wide survey and expression-profiling analysis further identified a set of $N A C$ genes involved in adaptation to drought ${ }^{35}$. NAC proteins are important regulators in a wide range of developmental processes like cell wall biosynthesis, the formation of lateral roots, floral morphogenesis, development of shoot apical meristem, embryo development, and grain nutrient remobilization ${ }^{36-41}$.

In a recent study, we found that the expression of the cucumber gene CUC3 encoding a NAC protein, was downregulated by $>1000$-fold in a spontaneous $t b h$ (tiny branched hair) mutant as compared with its wild type, suggesting its possible involvement in cucumber fruit-trichome development ${ }^{42}$. In another study, a cucumber NAC gene that is a homolog of Arabidopsis AtNAC25 showed no expression in the tril (trichomeless) glabrous mutant, but it was highly expressed in its wild type, implying that this $N A C$ gene was affected by TRIL (CsGL3), a key gene for regulating trichome development ${ }^{43}$. In cucumber, fruit trichomes are also called spines, which together with the tubercles form the warty fruit trait ${ }^{42,44}$. The presence or absence, the size, number, and density of fruit spines are important fruit-quality traits affecting cucumber fruit economic value and commercial marketability ${ }^{42,44,45}$. For example, North China fresh market (Chinese Long) cucumbers have fruits with many trichomes and a dull surface appearance, whereas European greenhouse cucumbers often have smooth and glossy fruits (no visible warts and spines).

Despite the importance in cucumber breeding for external fruit quality, little is known about the molecular mechanisms of the initiation and development of fruit spines $^{44}$. Although transcriptomic data from cucumber trichome-related mutants have suggested important roles of some NAC TFs in trichome organogenesis and development ${ }^{42,43,46}$, a systematic characterization of NAC TFs in the cucumber genome is lacking. Therefore, in this study, we performed genome-wide identification and characterization of NAC genes in the cucumber genome. To investigate the potential functions of NAC TFs in cucumber trichome development, we examined the expression of all $N A C$ genes in wild-type plants and the $t b h$ mutant. We identified a set of NAC genes that represent targets for future studies of cucumber fruit spine development.

\section{Methods}

\section{Plant materials and hormone treatments}

The cucumber inbred line R1407 (WT) and $t b h$ mutant ${ }^{42}$ plants were grown in a greenhouse in the experimental field of the China Agricultural University (Beijing, China). Cultured practices were carried out according to recommended protocols. 
To examine the effect of hormones on the expression of CsvNAC genes and trichome development, WT fruits $(2 \mathrm{~d}$ before flowering) were sprayed with $5 \mathrm{ml}$ of $\mathrm{GA}_{3}(50 \mu \mathrm{M})$, IAA $(200 \mu \mathrm{M})$, Me-JA $(200 \mu \mathrm{M})$, or ethephon $(1 \mathrm{mM})$. In the ethephon treatment, the pots were sealed with aluminum foil to maintain the ethylene gas enriched atmosphere. Control plants were sprayed with $5 \mathrm{ml}$ of $\mathrm{H}_{2} \mathrm{O}$. Trichomes were collected from the fruits at $0,6,12,24$, and $36 \mathrm{~h}$ after treatment. There were three independent replicates for each treatment.

\section{Database search and sequence retrieval}

The sequences of the 9930 cucumber draft genome (version 2.0) were extracted from the Cucurbit Genomics database (http://www.cucurbitgenomics.org/), and Arabidopsis sequences from TAIR (http://www.arabidopsis. org/). The hidden Markov model (HMM) profile of the NAM domain (PF02365) was downloaded from the Pfam database (Pfam 29.0) (http://pfam.sanger.ac.uk/) to identify NAC genes in the cucumber genome using HMMER 3.0 software with an E-value cutoff of $1^{3}$. The predicted Arabidopsis NAC protein sequences (http://www. arabidopsis.org) were used as a query against the predicted cucumber proteome sequences to identify all cucumber NAC proteins. The Multiple Expectation Maximization for Motif Elicitation (MEME) program version 4.9.1 (http://nbcr-222.ucsd.edu/opal-jobs/) was used to identify motifs in the 91 CsvNAC protein sequences. MEME was run locally with the following parameters: number of repetitions--any, maximum number of motifs- -5 , and the optimum motif widths were constrained to be between 6 and 250 residues. In addition, the three fields (length, molecular weight, and isoelectric point) of each NAC protein were predicted by the online program ExPasy (http://www.expasy.org/tools/).

\section{Phylogenetic analysis}

The MEGA 5 software package (http://www. megasoftware.net) was used for the construction of unrooted phylogenetic trees using the neighbor-joining $(\mathrm{NJ})$, minimal evolution (ME), and maximum parsimony (MP) methods, and bootstrap tests were carried out with 1000 replicates. The pairwise gap-deletion mode was used to ensure that the more divergent $\mathrm{C}$-terminal domains could contribute to the topology of the NJ tree ${ }^{3}$.

\section{Chromosomal location and analysis of the relationship of homologous $A$. thaliana members}

The nucleotide sequences of all cucumber $N A C$ genes were further used as a query for NCBI BLASTn (http:// blast.ncbi.nlm.nih.gov/Blast.cgi) searches against the cucumber chromosomes to determine their locations. Mapping was then conducted using the MapInspect program (http://mapinspect.software.informer.com/ $)^{3}$.
Orthomcl (http://orthomcl.org/orthomcl/) was used to perform relationship analyses of homologous members between cucumber and Arabidopsis ${ }^{47}$. The results were formatted for display using the Circos software (version 0.69, http://circos.ca/sofware/download/circos//48.

\section{Exon/intron structure analysis}

The exon/intron arrangement of the NAC genes was illustrated with the gene structure display server (GSDS) program (http://gsds.cbi.pku.edu.cn/) using both genomic DNA sequences and the corresponding coding sequences. The $5^{\prime}$ untranslated region (UTR) sequences were removed to allow better visualization and comparison.

\section{RNA isolation and quantitative real-time PCR (qPCR) analysis}

Roots, tendrils, male and female flowers, stems, leaves, and fruits were collected from R1407 for total RNA extraction using the Column Plant RNA out kit (TIANDZ, China) following the manufacturer's instructions. Fruit samples were collected at $-2,0,8$, and $14 \mathrm{~d}$ post anthesis. Samples were treated with RNase-free DNase I (Promega, USA) and quantified spectrophotometrically. PrimeScript First-Strand cDNA Synthesis SuperMix (TaKaRa, Japan) was used for synthesis of first-strand cDNA. Primers used for qPCR were designed with Primer 5.0 and the actin gene was used as the internal standard in the quantitation of template $\mathrm{CDNA}^{42}$. The qPCR was performed using SYBR ${ }^{\circledast}$ Premix Ex Taq ${ }^{\mathrm{TM}}$ (TaKaRa, China) with the Applied Bio-systems 7500 realtime PCR system (ABI, USA). The $-2^{-\Delta \Delta C t}$ method was used to determine the relative fold differences in template abundance for each sample ${ }^{42}$. For semiquantitative RTPCR, the conditions were as follows: $10 \mathrm{~min}$ at $94{ }^{\circ} \mathrm{C}, 30 \mathrm{~s}$ at $94{ }^{\circ} \mathrm{C}, 30 \mathrm{~s}$ at $50^{\circ} \mathrm{C}$, and $30 \mathrm{~s}$ at $72^{\circ} \mathrm{C}$, followed by $7 \mathrm{~min}$ at $72{ }^{\circ} \mathrm{C}$. Each cycle was repeated 25 times. The RT-PCR products were separated using a $2.0 \%(\mathrm{~m} / \mathrm{v})$ agarose gel. The gene-specific primers are listed in Supplementary Table S1.

\section{Cis-element and miRNA target analysis}

To identify putative cis-acting regulatory elements in the promoter regions of the CsvNAC genes, nucleotide sequences of the $2-\mathrm{kb}$ upstream regions from the transcription start site (TSS) were retrieved from the 9930 V2.0 draft genome sequences. Promoter cis-element analysis was carried out using the cis-acting regulatory elements database (PLANTCARE) (http://bioinformatics. psb.ugent.be/webtools/plantcare/html/).

For miRNA target analysis, the mature miRNA sequences were downloaded from the miR-Base v20.0 (http://www.mirbase.org/) and RMRD (http:// bioinformatics.cau.edu.cn/PMRD/). The prediction was performed using the plant small RNA target analysis 
server (http://plantgrn.noble.org/psRNATarget/) with default settings.

\section{Scanning electron microscopy (SEM) analysis}

Fruits at the flowering day were treated with $1 \%$ osmium tetroxide vapor for $24 \mathrm{~h}$ and then air-dried for $72 \mathrm{~h}$. The samples were examined by a SEM as described previously ${ }^{49}$. Images were collected using a Hitachi S-3400N microscope (Hitachi, Japan).

\section{Results}

\section{Identification and phylogenetic analysis of NAC family members}

To identify cucumber NAC TF encoding genes, the amino acid sequence corresponding to the conserved DNA-binding domain of known NAC proteins was used as the query (PF02365) in BLAST searches of the 9930 cucumber reference genome ${ }^{50}$. Ninety-one putative NAC genes were identified; each gene was annotated as CsvNACOXX, where Cs is the genus and species initials (Cucumis sativus), $\mathrm{v}$ is the latest version (v2) of the cucumber database, and $X X$ is the sequential number of a particular gene in the genome. The identified genes encoded predicted proteins ranging from 202 to 654 AA (amino acids) with isoelectric point (pI) values ranging from 4.52 to 9.53 and molecular weights from 26.2 to $73.7 \mathrm{kDa}$. Subcellular location of these genes was predicted using an online analysis tool from Molecular Bioinformatics Center (http://cello.life.nctu.edu.tw/). Among the 91 NAC proteins, one each was predicted to be located in the plasma membrane (CsvNAC019) or chloroplast (CsvNAC061); two (CsvNAC001 and 041) were extracellular; four (CsvNAC049, 53, 86, and 87) cytoplasmic; and the rest were localized in the nucleus. Detailed information, including accession numbers, exon number, sequence length, and chromosome locations of all identified NAC proteins is provided in Supplementary Table S2.

In order to explore the evolutionary relationships among members of NAC TF families, a neighbor-joining tree based on cucumber and Arabidopsis NAC protein sequences was constructed (Fig. 1). All members from both species were divided into seven subgroups that were designated as NACI to NACVII, respectively. Group NACV constituted the largest clade with 28 cucumber NAC members followed by NACIV with 25 cucumber protein sequences. The smallest clade, NACVII, had no members from cucumber.

\section{Chromosomal locations of CsvNAC genes and their relationship with Arabidopsis orthologs}

The $91 N A C$ genes were distributed unevenly in the cucumber genome with 14, 9, 19, 9, 11, 20, and 9 on chromosomes 1 through 7, respectively (Supplementary Figures S1 and S2). Using Orthomcl, the CsvNAC genes were comparatively mapped with their Arabidopsis orthologs $^{44}$. Maximum orthology was observed between CsvNAC genes on cucumber chromosome 5 and Arabidopsis chromosome 1, and the CsvNAC genes on cucumber chromosome 6 showed 67\% orthology and colinearity with the NAC genes on Arabidopsis chromosome 5 (Fig. 2). These results suggested similar evolutionary trends and that diversification of the NAC family occurred prior to the divergence of cucumber and Arabidopsis.

\section{CsvNAC gene structures and conserved motifs}

To better understand the relationships between the structure and functions among cucumber $N A C$ genes, the exon/intron organization and conserved motifs were analyzed, which are shown in Figs. 2 and 3, respectively. In the resulting phylogenetic tree, all 91 NAC members were divided into 10 subclades (Fig. 3 ). It seems that the most closely related members had similar numbers of exons (Fig. 3). For example, all nine members in subgroup A2 had three exons, while the seven members in subgroup $\mathrm{C}$ had a varying number of 3-7 exons. Among the 26 members in groups D1 and D2, all except two had three exons (Fig. 3).

Ten conserved motifs were identified among the 91 CsvNAC proteins (Supplementary Figure S3). As expected, the most closely related members in the same subfamilies shared a common motif composition, which may be indicative of similar functions (Fig. 4). Motif 7 (blue box in Fig. 4) was observed in all CsvNAC proteins, and most of the predicted motifs were located at the $\mathrm{N}$-terminus, which contains the A-E subdomains (motif 1-7 and motif 9) that confer DNA-binding activity. These results are consistent with previous studies showing a connection between subfamilies and motifs $^{22}$.

\section{Cis-element analysis}

Many cis-elements were detected in the CsvNAC gene promoter regions including some involved in hormone signaling, such as auxin, ethylene (Eth), gibberellin (GA), abscisic acid (ABA), and salicylic acid (SA). Most of the cucumber $C s v N A C$ promoters contained the conserved CGTCA element, suggesting self-regulation, since this is a sequence known to be bound by NAC $\mathrm{TFs}^{35}$. Some promoters also contained several abiotic stress response elements. For instance, CsvNAC053, 085, and 088 had more than one EDR or LRT element, which are known to be related to drought and chilling stresses ${ }^{31}$. In six NAC genes (CsvNAC010, 041, 048, 053, 058, and 059), cis-element analysis also revealed HD-Zip I binding sites in their promoters, which are important for trichome development ${ }^{42,43}$. Additional information regarding cis-acting regulatory elements that are involved in meristem specific 


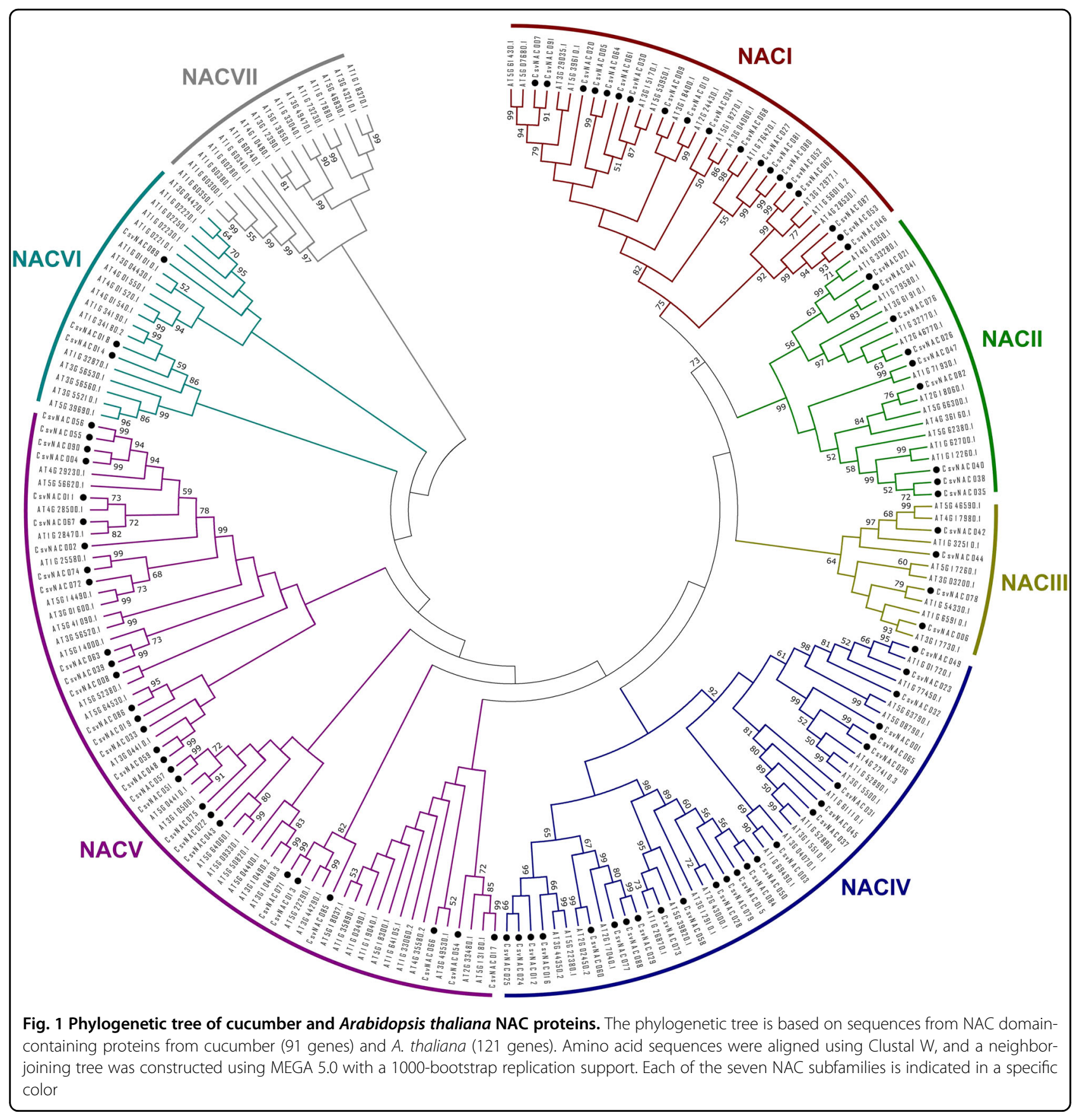

activation, methyl jasmonate (Me-JA)-responsiveness or differentiation of the palisade mesophyll cells, is provided in Supplementary Table S3.

\section{CsvNAC expression profiles in different organs in wild type and tbh mutant plants}

To gain insight into the temporal and spatial transcription patterns and putative functions of the CsvNAC genes in cucumber growth and development, we examined the expression patterns of all $91 N A C$ genes in the root, stem, leaf, tendril, male flower, female flower, and fruit with qRT-PCR (Fig. 5). Organ specific expression of different $N A C$ genes was obvious. For example, 11 genes (CsvNAC047, 048, 049, 054, 055, 058, 071, 072, 081, 082, and 088) were highly expressed in the stem, while 12 (CsvNAC020, 021, 022, 024, 027, 028, 029, 032, 033, 035, 038, and 078) were specifically expressed in the fruit. Similarly, small clusters of genes were specifically expressed in female flowers or leaves (Fig. 5, Supplementary Table S4). 


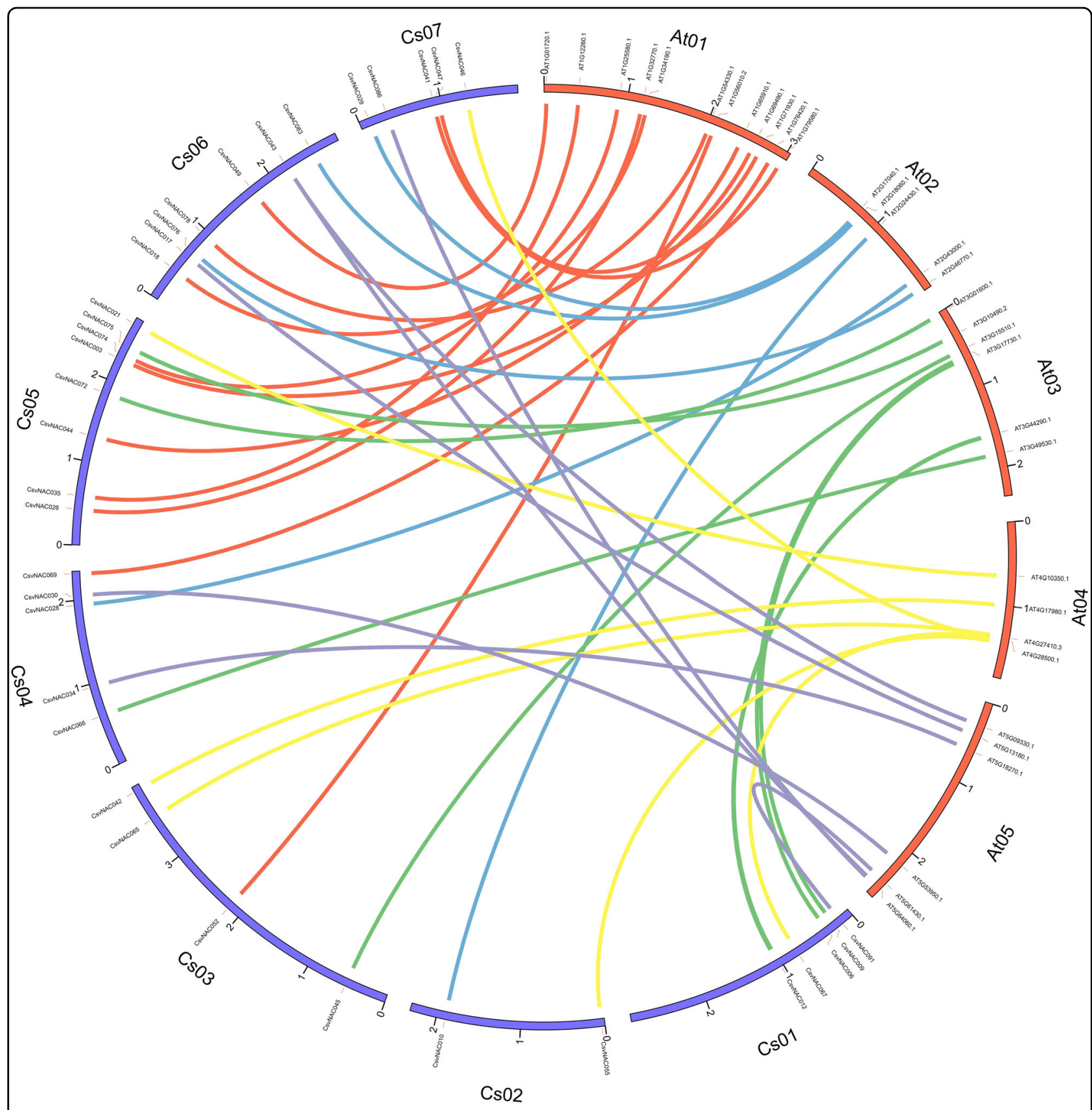

Fig. 2 Analysis of homologous member relationships between cucumber and A. thaliana. Orthomcl software was used to perform homologous member relationship analysis between cucumber and $A$. thaliana. The outer blue lines show the cucumber chromosomes and the red lines represent the A. thaliana chromosomes. Homologous cucumber and A. thaliana members are connected with different colored curved lines

We examined expression of CsvNAC genes in fruits at four development stages of both wild-type cucumber (R1407) and the tbh mutant (Fig. 6, Supplementary Table S5). Except for CsvNAC003, 045, 047, 051, 054, 057, 072, 075, and 079, most other CsvNAC genes exhibited similar expression profiles at Stages I ( $2 \mathrm{~d}$ before flowering) and II (The day of anthesis) (Fig. 6). It is noted that at stage III ( $8 \mathrm{~d}$ after flowering). Interestingly, CsvNACO69 exhibited high and slightly high expression in R1407 and the $t b h$ mutant, respectively only at the $8^{\text {th }}$ day post anthesis. Moreover, the expression level of other two genes, CsvNAC081 and CsvNAC049 were obvious increased at Stage III in R1407. One cluster of CsvNAC genes showed dramatic increase in their expression at stage IV, which was $14 \mathrm{~d}$ post anthesis. Since the spines were well developed by this time ${ }^{42}$, likely, they do not 


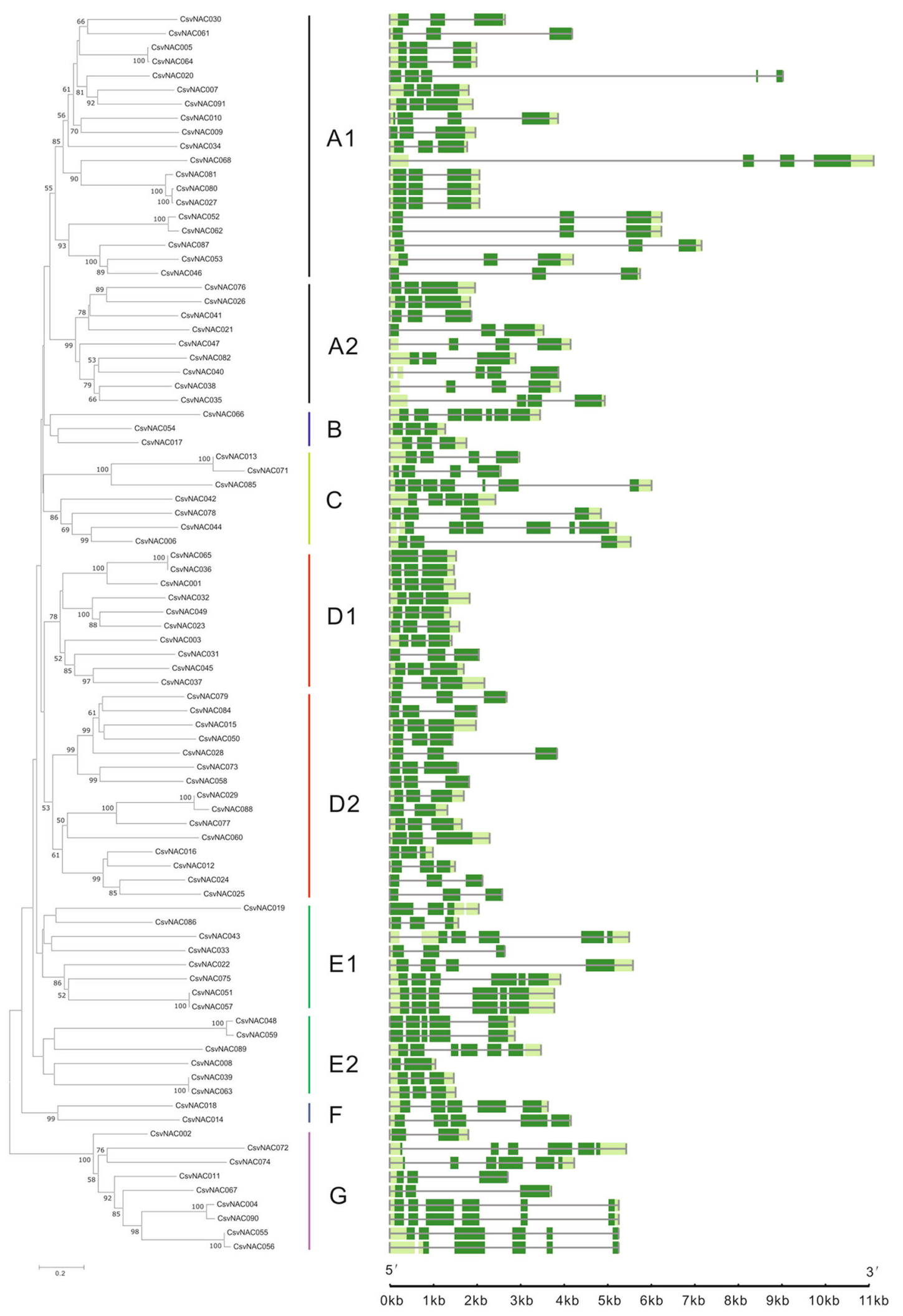

Fig. 3 Phylogenetic analysis and exon-intron structure of the CsvNAC genes. a The phylogenetic tree was generated using the neighborjoining (NJ) method implemented in the MEGA 5.0 software with the JTT model and the pairwise gap-deletion option. The bootstrap analysis was conducted with 1000 iterations. b The CSVNAC exon-intron distribution. The green bars indicate the exons, and the black lines indicate the introns 


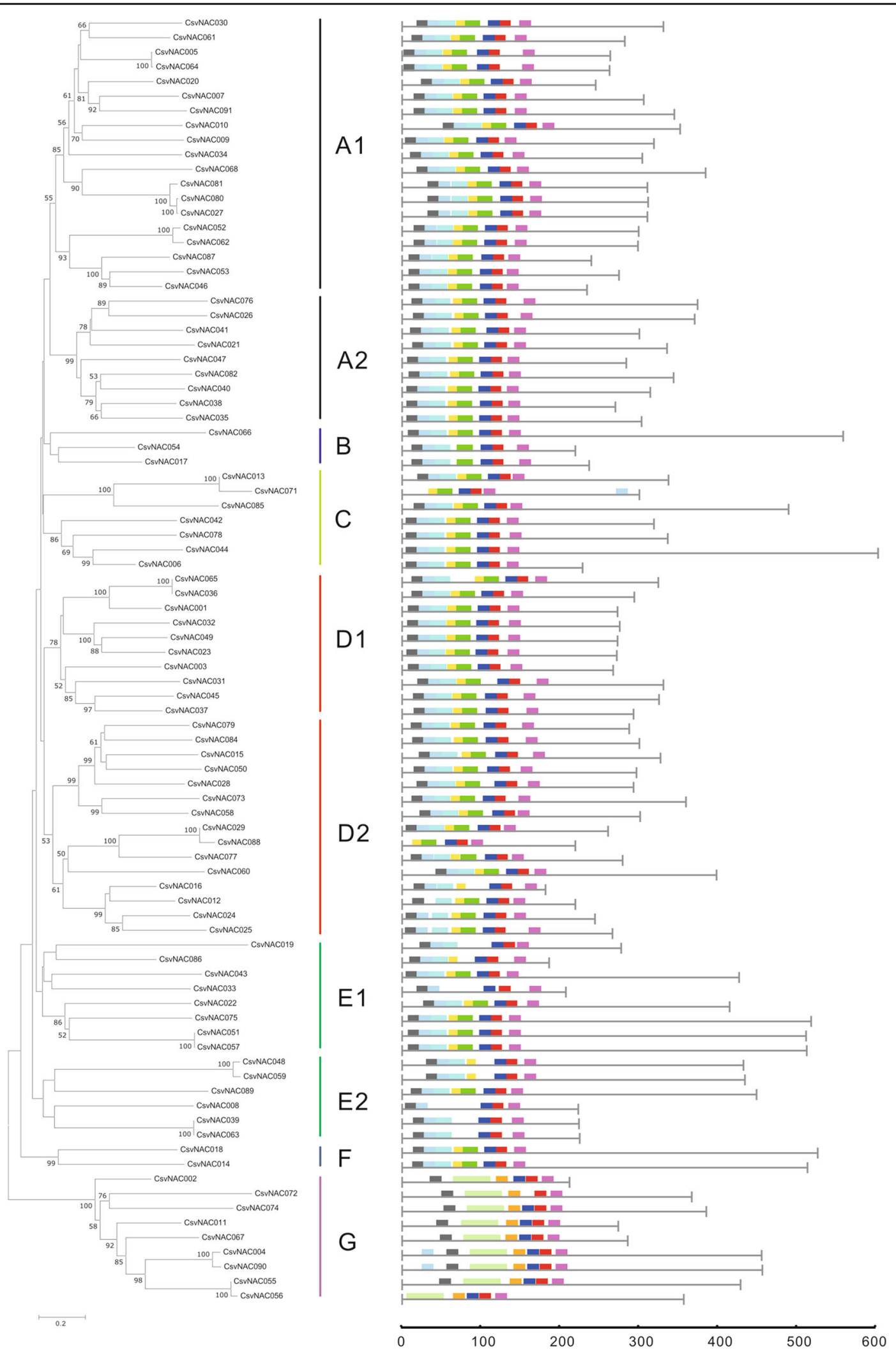

Fig. 4 Phylogenetic analysis and conserved motifs of the CsvNAC genes. a The phylogenetic tree was generated using the Neighbor-Joining (NJ) method implemented with MEGA 5.0 software and the pairwise gap-deletion option. The bootstrap analysis was conducted with 1000 iterations. b The motif composition of the cucumber NAC genes. Each color represents a specific motif 


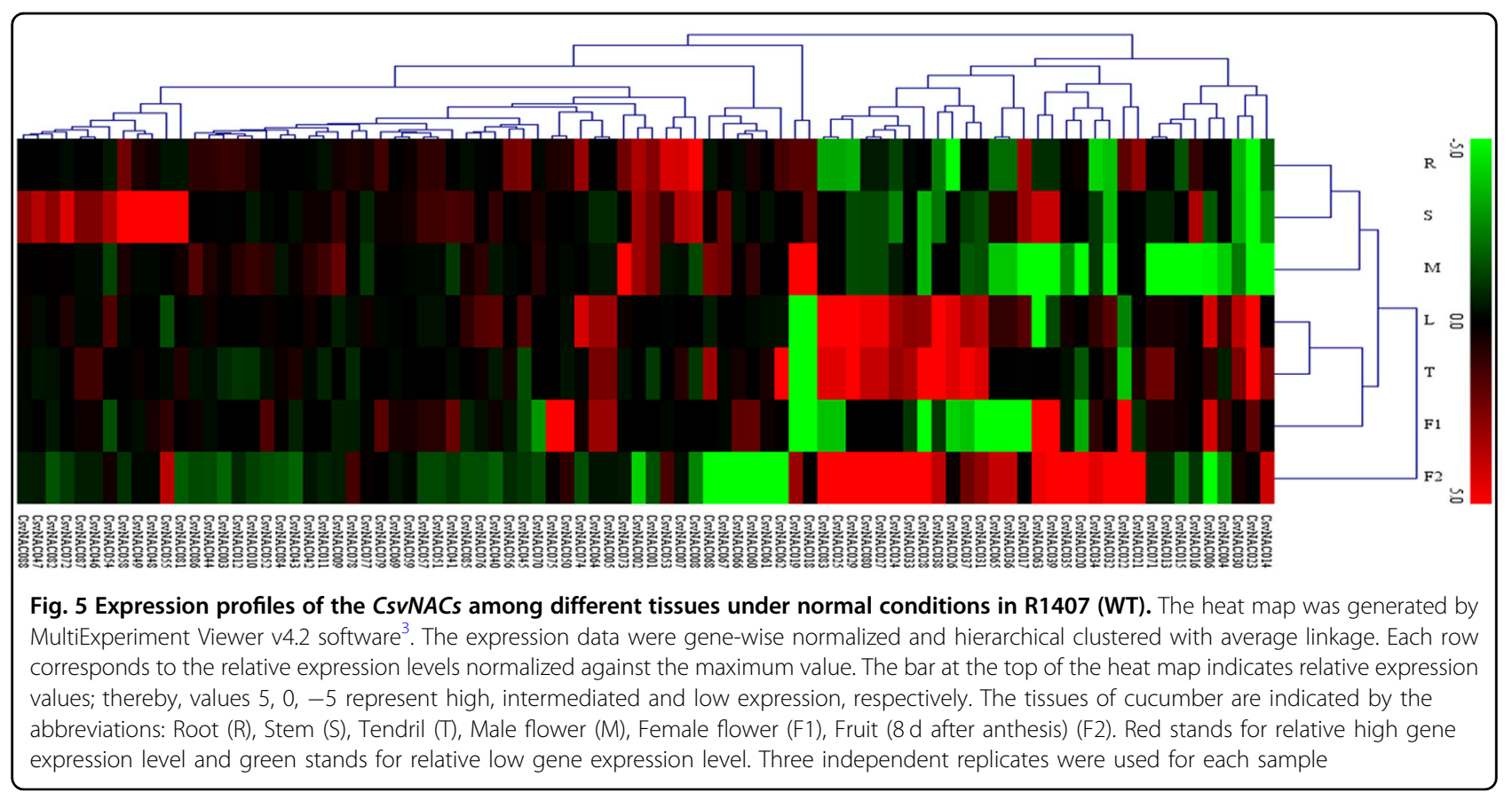

have any direct link with spine initiation or development (Supplementary Table S5).

\section{CsVNAC gene expression during trichome development in response to hormone treatments}

The effects of phytohormones, such as GA, IAA, Me-JA, and Eth, on trichome proliferation and development have been well documented ${ }^{26,51}$. To understand the effects of hormones on trichome development, we examined trichome density dynamics in response to treatments with GA, IAA, Me-JA, and Eth. We found that application of these hormones stimulated trichome formation and increased trichome density on the fruits (Fig. 7a). The spine density on treated fruits was significantly higher than that in the control (Fig. 7b) suggesting GA, IAA, Eth, and Me-JA may all promote trichome initiation. To further determine whether CsvNAC genes responded to hormone related signaling, cis-element analysis in the $2 \mathrm{~kb}$ promoter region of each gene was conducted (Supplementary Table S6). Motifs related with GA, IAA, Me-JA, and Eth in the promoters were identified and their corresponding transcript abundance was measured following treatments with different hormones. Application of all phytohormones significantly upregulated the expression of $N A C$ genes in fruit spines (Fig. 8). For example, upon GA3 application, the expression of most CsvNAC genes was upregulated peaking at $6-24 \mathrm{~h}$ post-treatment, followed by a sharp decline to the pre-treatment level, or to a level that was lower than in the control (Fig. 8a, Supplementary Table S7). Similarly, a substantial increase in the expression of all selected CsvNAC genes was observed 12 or $24 \mathrm{~h}$ post-treatment with Eth, with the exception of CsvNAC30, 031, 054, 070, and 088, all of which were downregulated (Fig. 8b, Supplementary Table S7). However, none of these genes showed a continuing elevated expression after $24 \mathrm{~h}$ of treatment. In a few cases, changes in the expression were observed following treatment with IAA. For example, CsvNAC020 and CsvNAC051 showed their highest transcript levels at $6 \mathrm{~h}$ post-application, and CsvNAC065 reached its peak of expression at $24 \mathrm{~h}$ after treatment. In general, a slight increase in expression of selected genes was observed $24 \mathrm{~h}$ after treatment (Fig. 8c, Supplementary Table S7). Me-JA induced expression of most CsvNAC genes after 24-36 h treatment, with the exception of CsvNACOO2 and CsvNAC074 (Fig. 8d, Supplementary Table S7). In contrast, CsvNAC032, CsvNAC033, and CsvNAC035 showed decreased transcript levels $6-12 \mathrm{~h}$ treatment, while the expression of CsvNAC072 and CsvNAC071 peaked at $6 \mathrm{~h}$ post Me-JA treatment and then declined. Taken together, exogenous hormones may affect CsvNACs expression so as to regulate trichome formation in cucumber.

\section{CsvNAC miRNA targets and gene ontology (GO) annotation}

Micro-RNAs (miRNAs) play roles in posttranscriptional gene regulation by either cleaving mRNA transcripts or repressing translation ${ }^{41}$. To assess the involvement of miRNAs in regulating the expression of CsvNAC genes, putative miRNA targets were determined in the 91 CsvNAC genes. Twelve were found to be the targets of 13 known cucumber miRNAs (Supplementary Table S6 and Supplementary Table S8). CsvNAC091 and 


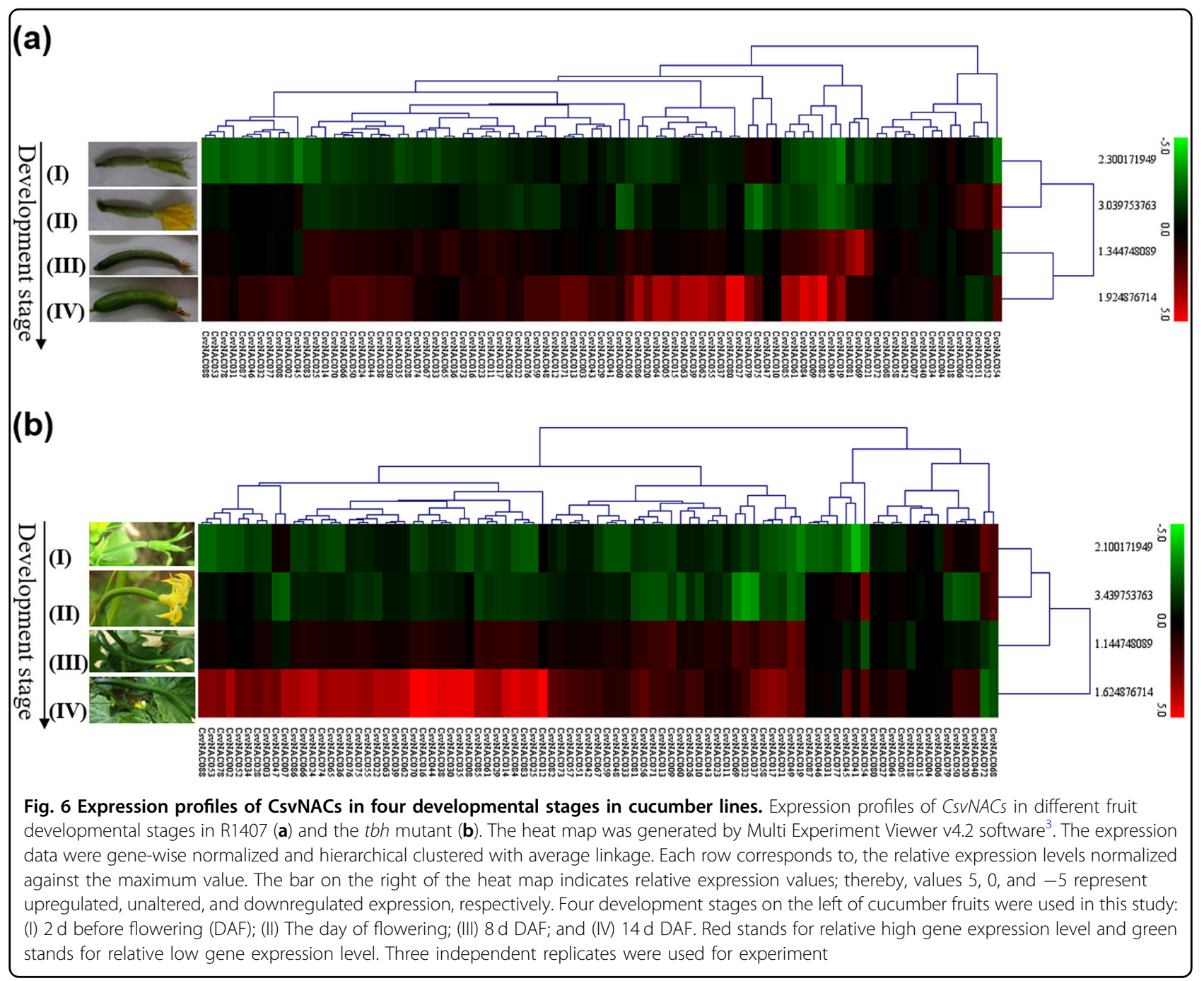

CsvNAC075 were each predicted to be the targets of 2 miRNAs (miR-164c and miR-164e-5p, or miR-169 and miR-169n, respectively). MiR-164a was predicted to target CsvNAC064, CsvNAC020, and CsvNAC005, and miR$164 \mathrm{~b}$ to target CsvNAC062, CsvNAC061, and CsvNAC052. These CsvNAC targets belonged to Subclass A1. In order to better understand the characteristics of these miRNA targets, GO enrichment analysis was performed using Blast2Go (https://www.blast2go.com/). This analysis suggested participation of the 12 CsvNAC proteins in diverse biological processes (Supplementary Table S8). Of the 4 categories of biological processes defined by Blast2Go, the CsvNAC proteins were predicted to function predominantly in 'developmental processes' (75\%) (Supplementary Table S8), followed by 'multicellular organismal processes' and 'cellular processes'. Cellular component prediction further suggested that 10 out of the 12 proteins are localized to the nucleus, and molecular function analysis suggested that those NAC proteins have nucleic acid binding TF activity (Supplementary Table S8).

\section{Discussion}

In this study, we performed a comprehensive analysis of

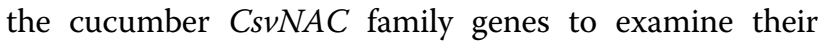
potential functions in fruit-trichome development. The 91 full-length NAC family proteins could be clustered into 6 subfamilies (NACI -VI), which was in contrast with 7 of Arabidopsis NAC proteins; no CsvNAC members were in the small NACVII clade with 16 Arabidopsis NAC members (Fig. 1). This may suggest functional divergence of some NAC genes between the two species. There were significantly more exons in members of $C$ and $G$ subfamilies (4-7) than in the D1 and D2 subfamilies (2-3). It has previously been reported that the rate of intron loss is faster than the rate of intron gain after segmental duplication in rice NAC genes ${ }^{52}$. Thus, it is likely that subfamilies $\mathrm{C}$ and $\mathrm{G}$ may contain more primitive genes, from 

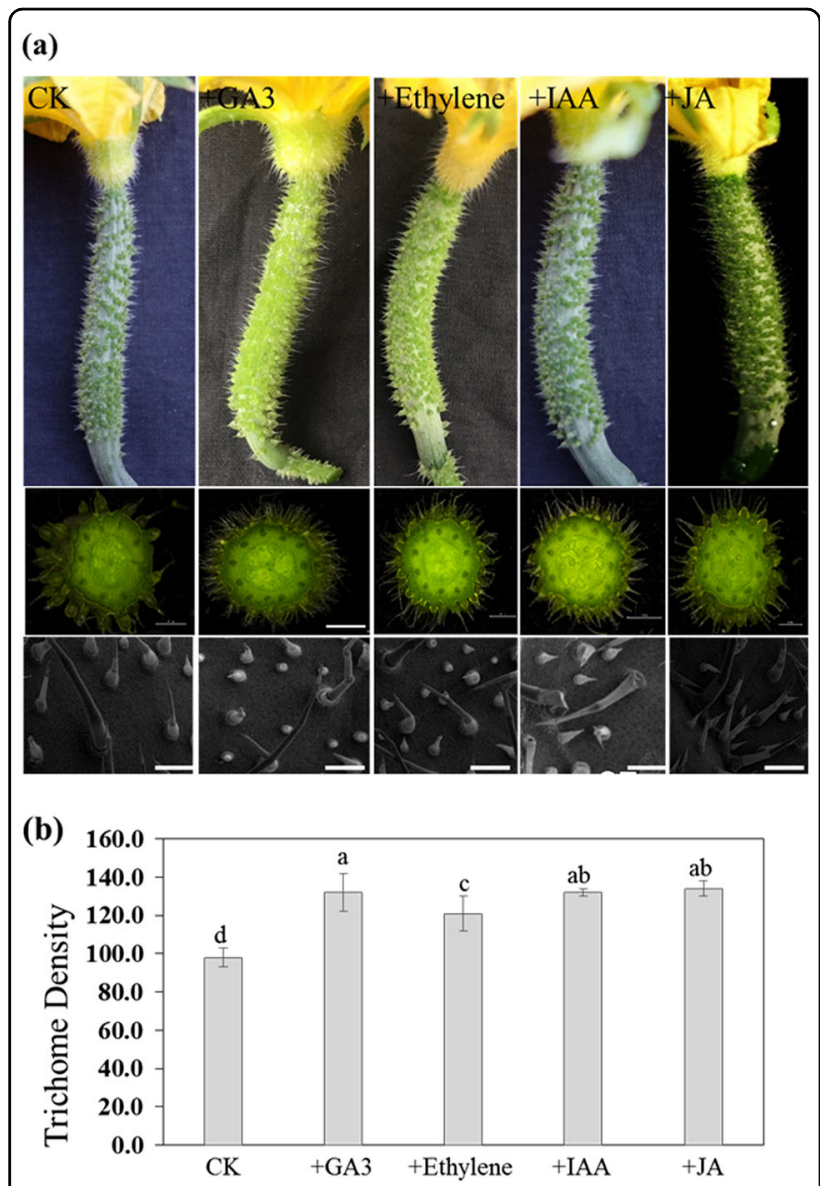

Fig. 7 Hormone treatments increase trichome numbers in cucumber fruit. a Fruits were either grown for $3 \mathrm{~d}$ under control treatment (wt) or for $3 \mathrm{~d}$ under $50 \mu \mathrm{M}$ GA3, 1 mM ethylene, $200 \mu \mathrm{M}$ IAA, and $200 \mu \mathrm{M}$ Me-JA. Scanning electron micrograph of trichomes stimulated after hormone treatments, showing a high density of trichomes. Scale bar $=200 \mu \mathrm{m}$. $\mathbf{b}$ Trichome numbers vary more in hormone treated than in those grown in the control. For each treatment, 20 whole fruit were examined. Mean trichome numbers $( \pm \mathrm{SD})$ are shown

which NAC genes in other clusters were derived. This phenomenon has also been reported in other species, such as Vitis vinifera and Manihot esculenta ${ }^{53}$. Conserved motif analysis revealed that almost all the CsvNAC proteins contained canonical A to E subdomains. In addition, each subfamily had other common motifs, while some subfamilies also harbored specific motifs (Fig. 4). Consistent with studies in Arabidopsis, rice, grapevine and cassava, our results suggest that CsvNAC TFs are evolutionarily conserved ${ }^{16-19,53,54}$.

To identify NAC genes playing possible roles in fruit spine development, we examined CsvNAC gene expression in ovaries/fruits from $2 \mathrm{~d}$ before flowering to $14 \mathrm{~d}$ post anthesis in R1407 and the spontaneous tbh trichome mutant (Fig. 6). We identified 12 CsvNAC genes differentially expressed in the $t b h$ mutant and R1407 from stages I to III. Based on our previous work, stages I and II are corresponding to spine initiation and formation; fruit spines mature at stage $\mathrm{III}^{42}$. Six genes, CsvNAC051, 057,003, 047, 075, and 045 showed higher expression in the wild type (R1407) than in the tbh mutant suggesting they may play positive regulatory roles in fruit spine development (Fig. 6). The expression pattern of some of these genes was consistent with previous studies ${ }^{42}$. For example, CsvNACOO3 was not expressed in the glabrous tril mutant, but highly expressed in the wild-type background, suggesting its involvement in trichome development ${ }^{43}$. In the present study, this gene also exhibited decreased expression in the $t b h$ mutant as compared with the WT (Fig. 6). It's homolog genes in cotton, GhNAC31, 49,73 , and 77 exhibit significantly higher expression at 10 $\mathrm{d}$ post anthesis (DPA) and were proposed to be involved in the early elongation phase of fiber development ${ }^{3}$. On the other hand, the three genes, CsvNAC079, CsvNAC072, and $C s v N A C 054$ were highly expressed in $t b h$ at stages I and II indicating their possible negative regulatory roles in spine development. It is worth to note that CsvNAC069 had and low expression in R1407 and in the tbh mutant only at the $8^{\text {th }}$ day post anthesis, which may suggest that CsvNAC069 plays its role in spine maturation. A cluster genes showed differential expression at stage IV, which may indicate their involvement in senescence of spines ${ }^{34}$. To summarize, our expression profiling of these CsvNAC genes provide us a global picture on their roles in spine initiation and development.

Plant hormones such as GA, Me-JA, and IAA are also known to influence trichome development ${ }^{51,54,55}$. A recent study found that GA distribution in Arabidopsis leaf mesophyll affected epidermis cell fate specification and promoted trichome formation ${ }^{55}$. Me-JA and its derivatives function as key signaling molecules in trichome formation; exogenous Me-JA causes an increase in the number of leaf trichomes in Arabidopsis ${ }^{51}$. The auxin response factor $(A R F)$ genes are involved in auxin (IAA) signaling transduction. In SlARF3 RNA interference (RNAi) lines in tomato, SlARF3-downregulated plants exhibited reduced density of types I, V, and VII trichomes on the leaves, which indicated the important roles of IAA transduction in the formation of trichomes in tomato ${ }^{54}$. Notably, we also found application of those hormones stimulated trichome formation and increased trichome density on the fruits (Fig. 7), suggesting GA, IAA, and MeJA may all promote trichome initiation. Previous work focused on plant leaf trichomes ${ }^{51,55}$. Here we found that GA, IAA, and Me-JA also play a vital role on the cucumber fruit spine development. In response to treatments with 4 hormones ost CsvNAC genes showed first increased then decreased expression pattern (Fig. 8), suggesting that most of these CsvNAC genes might have 


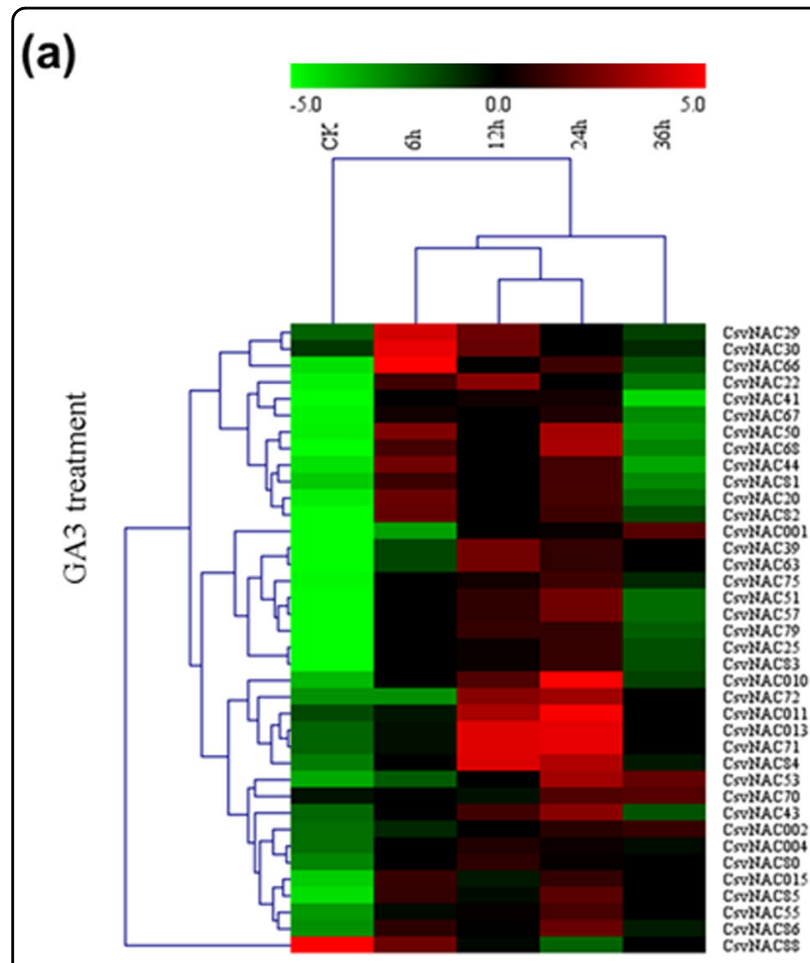

(b) (c)

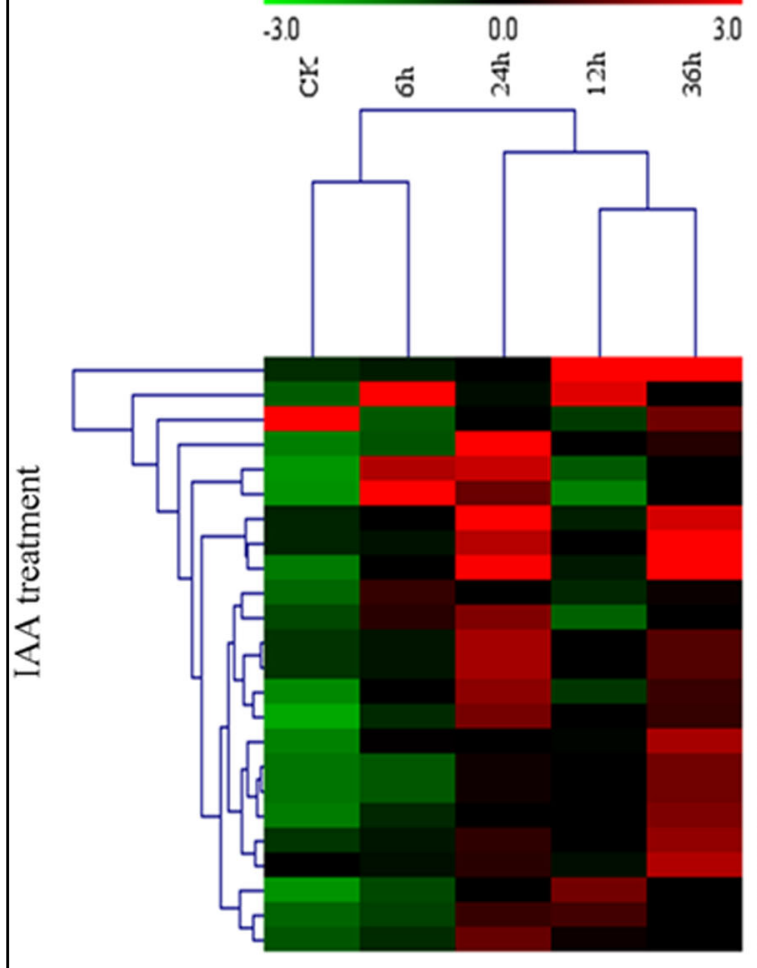

CsvNACO73 CsviACO20 CSNIACO40 Csviacoss CSvNACOO3 CsviACO21 CsvinaC031. Csviaco28 CSNIACOS2 CsNACO23 CsvNACO43 Csvinacos! CswNACOS7 Csvinac029 CsNNACOS9 CsvinaCO39 Csvinacoos CsvinaCOS4 CsvinaC071 CsvinaCOS1 CsNiACOT2 Csviac070 Cswiaco74 CSNNACOS?

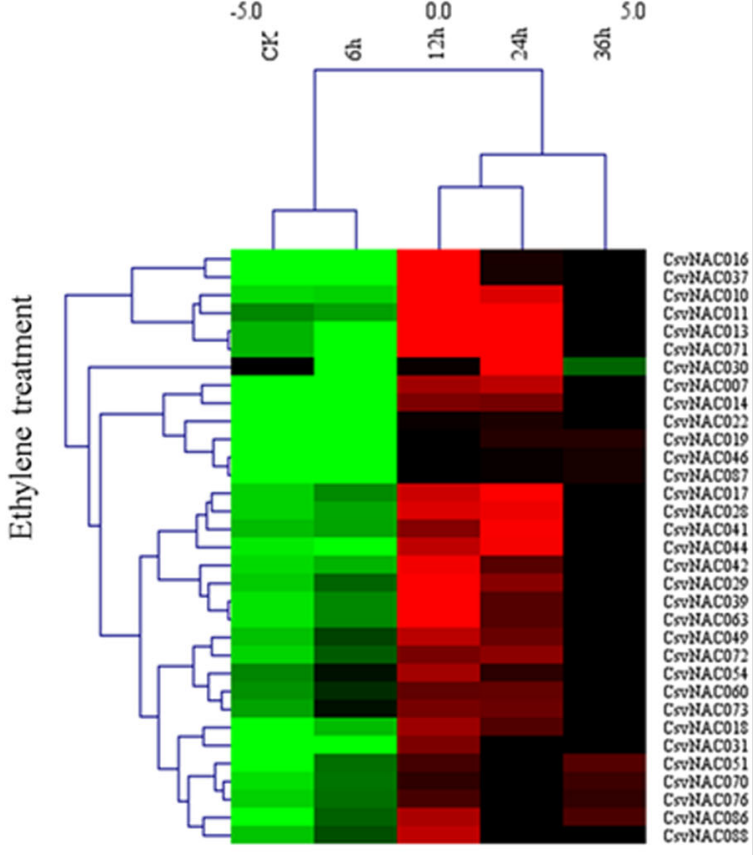

(d)
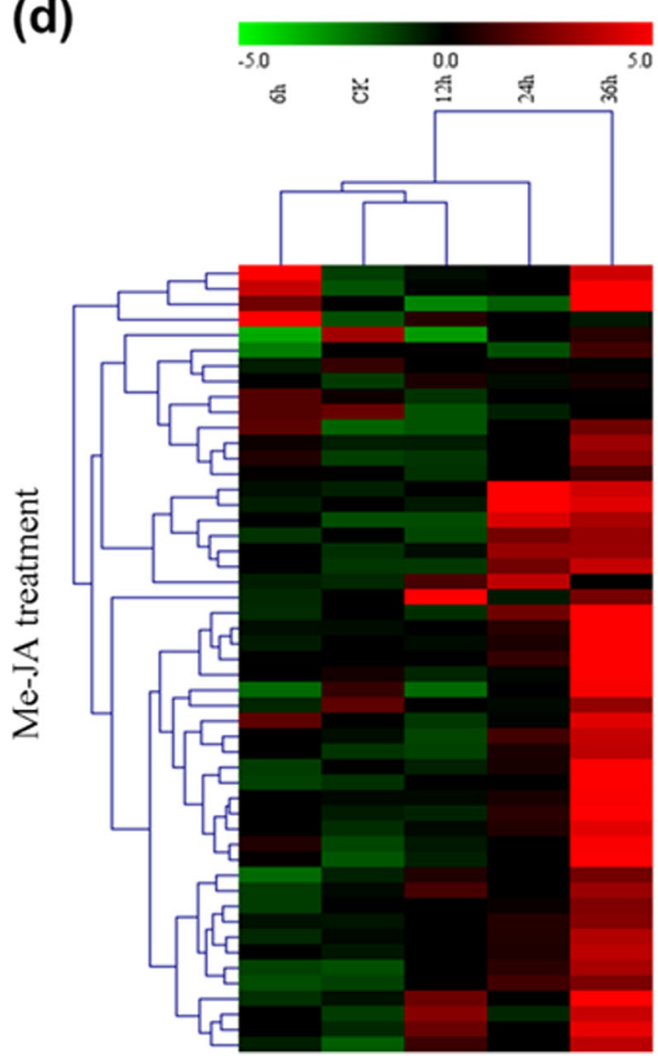

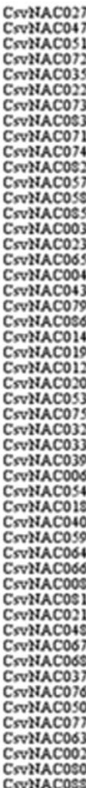

Fig. 8 (See legend on next page.) 
(see figure on previous page)

Fig. 8 Expression profiles of CsvNAC transcription following hormone treatments with Gibberellin (GA3), Ethylene (Eth), Auxin (IAA) and Jasmonic acid (Me-JA) in R1407. The heat map was generated by Multi Experiment Viewer v4.2 software ${ }^{3}$. The expression data were gene-wise normalized and hierarchical clustered with average linkage. Each row corresponds to, the relative expression levels normalized against the maximum value. The bar on the right of the heat map indicates relative expression values; thereby, values 5,0 , and -5 represent upregulated, unaltered, and downregulated expression in (a,b, d), respectively; 3,0, and-3 show high, intermediate, and low expression in (c), respectively. The CsVNAC genes are listed on the right of the expression array under GA3 (a), Ethylene (b), IAA(c), and Me-JA (d). Red stands for relative high gene expression level and green stands for relative low gene expression level. Three independent replicates were used for this experiment

GA, Eth, IAA, and Me-JA responsive elements, which may be due to crosstalks among the four hormone signaling pathways. However, some other genes, (e.g., CsvNAC007 and CsvNAC032) responded specifically to treatments with Eth and JA, respectively, indicating they may contain Eth and JA response elements and involvement of Eth and JA in inducing trichome development through the regulation of these TFs (Fig. 8). Indeed, most cucumber CsvNAC gene promoters contained the conserved AAACAGA (Gibberellin-responsive), CGTCA (Me-JA responsive), ATTTCAAA (Ethylene-responsive), and AACGAC (Auxin-responsive) elements (Supplementary Table S3), which can explain why treatments with these hormones caused CsvNACs transcriptional changes. Thus, these data suggested a connection among CsvNACs function, hormones signaling and spine development.

miRNAs are known to be involved in many aspects of plant development, but there were few reports on NAC miRNA targets specifically involved in plant trichome development. Here we found 12 CsvNAC genes that were potentially the targets of 13 miRNAs (Supplementary Table S6 and Supplementary Table S8). Most of the targets belonged to subclass A1, in which several trichome development related genes are located, indicating that the miRNAs identified here may be helpful in better understanding the transcriptional regulation of genes involved in this process. GO analysis further indicated that most of the target genes are involved in developmental processes (Supplementary Table S8), followed by multicellular organismal processes.

In summary, we identified $91 N A C$ genes in the cucumber genomes and examined their key structural features. The phylogenetic relationships among these $N A C$ genes were consistent with their exon/intron structurem, as well as the distribution of conserved domains. We identified tissue-specific, fruit developmentdependent differentially expressed CsvNAC genes in the tbh mutant, and its wild-type R1407 cucumber. Most genes were responsive to treatments by 4 hormones. A significant portion of the CsvNAC genes displayed preferential expression in fruit spines. Moreover, we identified 13 known cucumber miRNAs that target CsvNACs. The information obtained from this study provides new insights into potentials roles of $C s N A C$ genes in regulating cucumber fruit spine development. Those differentially expressed genes and miRNAs predicted from this analysis should be very valuable for functional analysis of CsNAC genes. This work also has practical value in developing cultivars adapting to different market requirements.

\section{Acknowledgements}

This work was supported by the National Natural Science Foundation of China (31672159), the National Key Research and Development Program of China (2016YFD0101705), the Project of Beijing Agricultural Innovation Consortium (BAIC01-2017) to HZR, and the Beijing Municipal Natural Science Foundation (6184043) to XWL.

\section{Author details}

${ }^{1}$ Beijing Key Laboratory of Growth and Developmental Regulation for Protected Vegetable Crops, College of Horticulture, China Agricultural University, 100193 Beijing, P. R. China. ${ }^{2}$ Department of Horticulture, USDA-ARS, Vegetable Crops Research Unit, University of Wisconsin-, Madison, WI 53706, USA

\section{Conflict of interest}

The authors declare that they have no conflict of interest.

Supplementary Information accompanies this paper at https://doi.org/ 10.1038/s41438-018-0036-z.

Received: 4 September 2017 Revised: 16 March 2018 Accepted: 21 March 2018

Published online: 01 June 2018

\section{References}

1. Chapman, E. J. \& Estelle, M. Mechanism of auxin-regulated gene expression in plants. Annu. Rev. Genet. 43, 265-285 (2009).

2. Hu, R. et al. Comprehensive analysis of NAC domain transcription factor gene family in Populus trichocarpa. BMC Plant Biol. 10, 145 (2010).

3. Shah, S. et al. Molecular cloning and functional analysis of NAC family genes associated with leaf senescence and stresses in Gossypium hirsutum L. Plant Cell. Tiss. Organ Cult. 117, 167-186 (2014).

4. Guo, Y. \& Gan, S. AtNAP, a NAC family transcription factor, has an important role in leaf senescence. Plant J. 46, 601-612 (2006).

5. Wang, L. et al. Genome-wide survey of pseudogenes in 80 fully re-sequenced Arabidopsis thaliana accessions. PLoS One. 7(12), e51769 (2012).

6. Yamasaki, K. et al. Structures and evolutionary origins of plant-specific transcription factor DNA-binding domains. Plant Physiol. Biochem. 46, 394-401 (2008).

7. Wang, Z. et al. Genome-wide analysis of the basic leucine zipper (bZIP) transcription factor gene family in six legume genomes. BMC Genom. 16, 1053 (2015).

8. Kavas, M. et al. Genome-wide characterization and expression analysis of common bean bHLH transcription factors in response to excess salt concentration. Mol. Genet. Genom. 29, 129-143 (2016).

9. Souer, E., van Houwelingen, A., Kloos, D., Mol, J. \& Koes, R. The no apical meristem gene of Petunia is required for pattern formation in embryos and flowers and is expressed at meristem and primordia boundaries. Cell $\mathbf{8 5}$, 159-170 (1996). 
10. Aida, M., Ishida., T., Fukaki., H., Fujisawa, H. \& Tasaka, M. Genes involved in organ separation in Arabidopsis: an analysis of the cup-shaped cotyledon mutant. Plant. Cell. 9, 841-857 (1997).

11. Gong, W. et al. Genome-wide ORFeome cloning and analysis of Arabidopsis transcription factor genes. Plant Physiol. 135, 773-782 (2004).

12. Xiong, $Y$. et al. Transcription factors in rice: a genome-wide comparative analysis between monocots and eudicots. Plant Mol. Biol. 59, 191-203 (2005).

13. Pinheiro, G. et al. Complete inventory of soybean NAC transcription factors: sequence conservation and expression analysis uncover their distinct roles in stress response. Gene 444, 10-23 (2009).

14. Huang, S. et al. Genome-wide analysis of WRKY transcription factors in Solanum Iycopersicum. Mol. Genet. Genom. 287, 495-513 (2012)

15. Hu, W. et al. Genome-wide identification and expression analysis of the NAC transcription factor family in cassava. PLoS One. 10, e0136993 (2015).

16. Cenci, A., Guignon, V., Roux, N. \& Rouard, M. Genomic analysis of NAC transcription factors in banana (Musa acuminata) and definition of NAC orthologous groups for monocots and dictos. Plant Mol. Biol. 85, 63-80 (2014).

17. Wang, N., Zheng, Y., Xin, H., Fang, L. \& Li, S. Comprehensive analysis of NAC domain transcription factor gene family in Vitis vinifera. Plant Cell. Rep. 32, 61-75 (2013)

18. Ernst, H. A., Olsen, A. N., Skriver, K., Larsen, S. \& Leggio, L. L. Structure of the conserved domain of ANAC, a member of the NAC family of transcription factors. EMBO Rep. 5, 297-303 (2004).

19. Olsen, A. N., Ernst, H. A., Leggio, L. L. \& Skriver, K. NAC transcription factors: structurally distinct, functionally diverse. Trends Plant. Sci. 10, 79-87 (2005).

20. Jensen, $M$. et al. The Arabidopsis thaliana NAC transcription factor family: structure-function relationships and determinants of ANAC019 stress signaling. Biochem. J. 426, 183-196 (2010).

21. Ooka, $\mathrm{H}$. et al. Comprehensive analysis of NAC family genes in Oryza sativa and Arabidopsis thaliana. DNA Res. 10, 239-247 (2003).

22. Chen, Q., Wang, Q., Xiong, L. \& Lou, Z. A structural view of the conserved domain of rice stress-responsive NAC1. Protein Cell 2, 55-63 (2011).

23. Lu, M. et al. A maize stress-responsive NAC transcription factor, ZmSNAC1, confers enhanced tolerance to dehydration in transgenic Arabidopsis. Plant Cell Rep. 31, 1701-1711 (2012).

24. Puranik, S., Sahu, P. P., Srivastava, P. S. \& Prasad, M. NAC proteins: regulation and role in stress tolerance. Trends Plant. Sci. 17, 369-381 (2012).

25. Fang, Y., You, J., Xie, K., Xie, W. \& Xiong, L. Systematic sequence analysis and identification of tissue-specific or stress-responsive genes of NAC transcription factor family in rice. Mol. Genet. Genom. 280, 547-563 (2008).

26. Xie, Q. et al. SINAT5 promotes ubiquitin-related degradation of NAC1 to attenuate auxin signals. Nature 419, 167-170 (2002).

27. Hao, Y. et al. Plant NAC-type transcription factor proteins contain a NARD domain for repression of Transcriptional activation. Planta 232, 1033-1043 (2010).

28. Seo., P. J., Kim, S. G. \& Park, C. M. Membrane-bound transcription factors in plants. Trends Plant. Sci. 13, 550-556 (2008).

29. Shen, H., Yin, Y., Chen, F., Xu, Y. \& Dixon, R. A. A bioinformatic analysis of NAC genes for plant cell wall development in relation to lignocellulosic bioenergy production. Bioenerg. Res. 2, 217-232 (2009).

30. Kim, S. et al. Genome-scale screening and molecular characterization of membrane-bound transcription factors in Arabidopsis and rice. Genomics 95 56-65 (2010).

31. Le, D. et al. Genome-wide survey and expression analysis of the plant-specific NAC transcription factor family in soybean during development and dehydration stress. DNA Res. 18, 263-276 (2011).

32. Nakashima, K., Takasaki, H., Mizoi, J., Shinozaki, K. \& Yamaguchi-Shinozaki, K. NAC transcription factors in plant abiotic stress responses. Biochem. Biophys. Acta 1819, 97-103 (2012).

33. Mao, $\mathrm{H}$. et al. A transposable element in a NAC gene is associated with drought tolerance in maize seedlings. Nat. Commun. 6, 8326 (2015)
34. Podzimska-Sroka, D., O'Shea, C., Gregersen, P. L. \& Skriver, K. NAC transcription factors in senescence: from molecular structure to function in crops. Plants $\mathbf{4}$, 412-448 (2015)

35. Shiriga, K et al. Genome-wide identification and expression pattern of drought-responsive members of the NAC family in maize. Meta Gene 2, 407-417 (2014)

36. Kikuchi, K. et al. Molecular analysis of the NAC gene family in rice. Mol. Gen. Genet. 262, 1047-1051 (2000).

37. Xie, Q., Frugis, G., Colgan, D. \& Chua, N. H. Arabidopsis NAC1 transduces auxin signal downstream of TIR1 to promote lateral root development. Genes Dev. 14, 3024-3036 (2000)

38. Mitsuda, N., Seki, M., Shinozaki, K. \& Ohme-Takagi, M. The NAC transcription factors NST1 and NST2 of Arabidopsis regulate secondary wall thickenings and are required for anther dehiscence. Plant Cell 17, 2993-3006 (2005).

39. Mitsuda, N. et al. NAC transcription factors, NST1 and NST3, are key regulators of the formation of secondary walls in woody tissues of Arabidopsis. Plant Cell 19, 270-280 (2007).

40. Larsson, E., Sitbon, F., Sundström, J. \& von Arnold, S. Expression of PaNAC01, a Picea abies CUP-SHAPED COTYLEDON orthologue, is regulated by polar auxin transport and associated with differentiation of the shoot apical meristem and formation of separated cotyledons. Ann. Bot. 110, 923-934 (2012).

41. Lan, Y. et al. Identification of novel MiRNAs and MiRNA expression profiling during grain development in indica rice. BMC Genom. 13, 264 (2012).

42. Chen, C. et al. Transcriptome profiling reveals roles of meristem regulators and polarity genes during fruit trichome development in cucumber (Cucumis sativus L.). J. Exp. Bot. 65, 4943-4958 (2014).

43. Zhao, J. et al. Transcriptome profiling of trichome-less reveals genes associated with multicellular trichome development in Cucumis sativus. Mol. Genet. Genom. 290, 2007-2018 (2015)

44. Liu, X. W., Bartholonew, E., Cai, Y. L. \& Ren, H. Z. Trichome-related mutants provide a new perspective on multicellular trichome initiation and development in cucumber (Cucumis sativus. L). Front. Plant Sci. 7, 1187 (2016).

45. Li, Y. H., Wen, C. L. \& Weng, Y. Q. Fine mapping of the pleiotropic locus $B$ for black spine and orange mature fruit color in cucumber identifies a $50 \mathrm{~kb}$ region containing a R2R3-MYB transcription factor. Theor. Appl. Genet. 126, 2187-2196 (2013)

46. Li, Q. et al. The identification of Cucumis sativus Glabrous 1(CSGL1) required for the formation of trichomes uncovers a novel function for the homeodomainleucine zipper I gene. J. Exp. Bot. 66, 2515-2516 (2015).

47. Li, L., Stoeckert, C. J. \& Roos, D. S. OrthoMCL: identification of ortholog groups for eukaryotic genomes. Genome Res. 13, 2178-2189 (2003).

48. Krzywinski, M. et al. Circos: an information aesthetic for comparative genomics. Genome Res. 19, 1639-1645 (2009)

49. Wang, W. et al. Cucumber CECRIFRUMI1 (CSCER1), which influences the cuticle properties and drought tolerance of cucumber, plays a key role in VLC alkanes biosynthesis. Plant Mol. Biol. 87, 219-233 (2015).

50. Huang, S. et al. The genome of the cucumber, Cucumis sativus L. Nat. Genet. 41, 1275-1281 (2009)

51. An., L., Zhou, Z., Yan, A. \& Gan, Y. Progress on trichome development regulated by phytohormones signaling. Plant Signal. Behav. 6, 1959-1962 (2011).

52. Liu, J., Chen, K., Zhang, Z., Chen, X. \& Wang, A. Effects of exogenous GA, Me-JA IAA, SA and KT on trichome formation in tomato. Acta Horti Sin. (Chin.) 43 2151-2160 (2016).

53. Nuruzzaman, M. et al. Genome-wide analysis of NAC transcription factor family in rice. Gene 465, 30-44 (2010).

54. Zhang, $X$. et al. Response gene SIARF3 plays multiple roles in tomato development and is involved in the formation of epidermal cells and trichomes. Plant Cell Physiol. 56, 2110-2124 (2015).

55. Greenboim-Wainberg, Y. et al. Cross talk between gibberellin and cytokinin: the Arabidopsis GA response inhibitor SPINDLY plays a positive role in cytokinin signaling. Plant Cell 17, 92-102 (2005). 